Agrotrópica 30(1): 43 - 48. 2018.

Centro de Pesquisas do Cacau, Ilhéus, Bahia, Brasil

\title{
BESOUROS ESCARABEÍDEOS (COLEOPTERA: SCARABAEIDAE) COLETADOS EM REMANESCENTE FLORESTAL EM RIO BRANCO, ACRE, BRASIL
}

\author{
Rodrigo Souza Santos ${ }^{1}$, Weidson Plauter Sutil', José Fernando Araújo de Oliveira ${ }^{2}$ \\ 'Embrapa Acre, Rodovia BR 364, km 14, CP 321, 69900-970, Rio Branco, AC, Brasil. rodrigo.s.santos@embrapa.br \\ ${ }^{2}$ União Educacional do Norte (UNINORTE), Alameda Hungria, 200, Jardim Europa, 69915-901, Rio Branco, AC. \\ plauter80@gmail.com; jfernando.a.o@hotmail.com
}

Besouros da família Scarabaeidae possuem grande importância ecológica, desde sua participação no ciclo de decomposição da matéria orgânica, no controle biológico da mosca-dos-chifres e na entomologia forense. Neste contexto, este trabalho teve como objetivo conhecer as espécies de besouros escarabeídeos em um remanescente florestal, localizado no município de Rio Branco, Acre. Durante o período de março a agosto de 2016 foram instaladas nove armadilhas do tipo "pitfall trap", com três tipos de atrativos alimentares (fezes humanas, coração bovino em decomposição e banana fermentada), dispostas em um triângulo equilátero, com 15 metros de lado. Foram realizadas duas coletas semanais (segundas e sextas-feiras), e as armadilhas eram recolhidas e substituídas por novas, incluindo os atrativos alimentares. O material foi triado no Laboratório de Entomologia da Embrapa Acre e os escarabeídeos preservados em álcool 70\%. Foram capturados um total de 764 espécimes, representados por cinco tribos, 12 gêneros e 40 espécies. Canthon quinquemaculatus, Canthon septemaculatus, Deltochilum carinatum, Deltochilum orbiculare, Dichotomius batesi, Phanaeus bispinus e Phanaeus camberforti representam primeiros registros de ocorrência para o estado do Acre. Pelo número de espécies capturadas, bem como pelos seus hábitos alimentares, conclui-se que o remanescente florestal possui baixo impacto antrópico.

Palavras-chave: Bioindicador, Fauna edáfica, Floresta tropical, Scarabaeoidea

\footnotetext{
Dung beetles (Coleoptera: Scarabaeidae) collected in forest remnant in the municipality of Rio Branco, Acre state, Brazil. Beetles of the family Scarabaeidae have great ecological importance, since its participation in the cycle of decomposition of organic matter, in the biological control of flies and in forensic entomology. In this context, the aim of this work was to know as species of Scarabaeidae beetles in a forest remnant, in the municipality of Rio Branco, Acre state, Brazil using pitfall traps, with three types of food attractions (human feces, bovine heart in decomposition and fermented banana). During the period from March to August 2016, two weekly collections were held (Mondays and Fridays), collecting the traps and replacing them with new ones, including food attractions. The material was screened in the Embrapa Acre's Laboratory of Entomology and the Scarabaeidae preserved in alcohol 70\%. A total of 764 specimens were collected, represented by five tribes, 12 genus and 40 species. The species Canthon quinquemaculatus, Canthon septemaculatus, Deltochilum carinatum, Deltochilum orbiculare, Dichotomius batesi, Phanaeus bispinus and Phanaeus camberforti are first records for Acre state. By the number of species captured, as well as by their eating habits, it is concluded that the forest remnant has low anthropic impact.
}

Key words: Bioindicator, Edaphic fauna, Tropical forest, Scarabaeoidea 


\section{Introdução}

A exploração de ambientes naturais com o propósito de expansão das diversas atividades humanas de produção, principalmente as de natureza agropecuária, provoca alterações na complexidade e estruturação dos diferentes habitats naturais e, consequentemente, acarretam em uma mudança na composição de espécies das comunidades animais anteriormente ali presentes (Borges, 2006). Essa prática tem como consequência a perturbação do equilíbrio dinâmico entre as espécies e o ambiente, podendo levar à extinção das espécies. A perda da biodiversidade ameaça a sustentabilidade de todo o sistema, com uma consequente redução dos serviços ambientais, tais como: controle de pragas, ciclagem de nutrientes e manutenção da estrutura do solo (Sampaio, 2010).

Uma forma de avaliar o grau de alteração ou fragmentação de um hábitat é mediante a utilização de um grupo de organismos considerados bioindicadores (Wink et al., 2005). Dentre os organismos bioindicadores, os insetos são considerados muito importantes na ecologia dos ecossistemas naturais, podendo ser utilizados em estudos de perturbação ambiental (Rosenberg et al., 1986).

A ordem Coleoptera é a maior dentre todas da Classe Insecta, e o número total de espécies varia de acordo com a opinião de diferentes autores, no entanto, segundo Slipinski et al. (2011), esta ordem possui cerca de 380.000 espécies conhecidas, representando $25 \%$ de todas as espécies de animais do planeta. Dentre estes, a família Scarabaeidae (superfamília Scarabaeoidea) se destaca como uma das principais, com aproximadamente 7.000 espécies conhecidas em nível mundial, das quais, cerca de 800 ocorrem no Brasil (Melo et al., 2009). Este grupo de besouros é detritívoro, promovendo a remoção e reingresso da matéria orgânica no ecossistema, aumentando a aeração do solo e prolongando a sua capacidade produtiva (Milhomem et al., 2003), podendo ainda ser considerados como indicadores de qualidade ambiental e de seus impactos em muitas regiões do mundo (Halffter e Favila, 1993).

A melhor maneira de se coletar coleópteros é com a utilização de iscas ou atrativos, independente do tipo de armadilha. No entanto, para a coleta de escarabeídeos, os quais são atraídos por fezes, carcaças e frutos em decomposição, mas não são atraídos por luz, as armadilhas de queda ou alçapões (pitfall) são as mais recomendadas (Favila e Halffter, 1997). A maioria das espécies utiliza excrementos, carcaças e frutas podres como recurso alimentar tanto na fase adulta quanto na larval (Halffter e Matthew, 1966). Portanto, a maior parte do grupo é facilmente amostrada através de armadilhas de queda iscadas com os recursos de que se alimenta (Lobo et al., 1988; Halffter e Favila, 1993).

Visto que, a fauna do solo exerce importante papel nos processos ecológicos do ecossistema, estudos sobre a composição e estrutura dessas comunidades são importantes para entender o seu funcionamento (Teixeira et al., 2009). Neste contexto e, levando em consideração que estudos dessa natureza ainda são muito escassos para o estado do Acre, este trabalho teve como objetivo conhecer as espécies de besouros escarabeídeos em um remanescente florestal, localizado no município de Rio Branco, visando contribuir para o aumento do conhecimento acerca das espécies de escarabeídeos ocorrentes no estado do Acre.

\section{Material e Métodos}

O estudo foi realizado em um remanescente florestal situado no campo experimental da Embrapa Acre (1001'49.8' S; 67\%41'00.5" W - altitude de 143 $\mathrm{m})$, no período de março a agosto de 2016, sendo realizadas duas coletas semanais (segundas e sextasfeiras), totalizando oito coletas mensais e um total de 48 coletas durante todo o período de amostragem.

O remanescente florestal é caracterizado como uma floresta ombrófila aberta, com área de aproximadamente 800 ha e faz fronteira com duas propriedades onde existem pastagens formadas. A fisionomia dessa floresta é predominantemente aberta com presença de tabocas (Guadua sp.), palmeiras e cipós. O clima é do tipo Aw (classificação de Köppen), ou seja, clima quente e úmido de monções, com uma estação seca bem diferenciada entre os meses de junho e outubro (Funtac, 1989; Oliveira, 1994; Oliveira e Braz, 1998).

$\mathrm{Na}$ área experimental foram instaladas nove armadilhas de queda do tipo "pitfall trap", dispostas em três triângulos de cinco metros de distância (três armadilhas por triângulo), formando um triângulo 


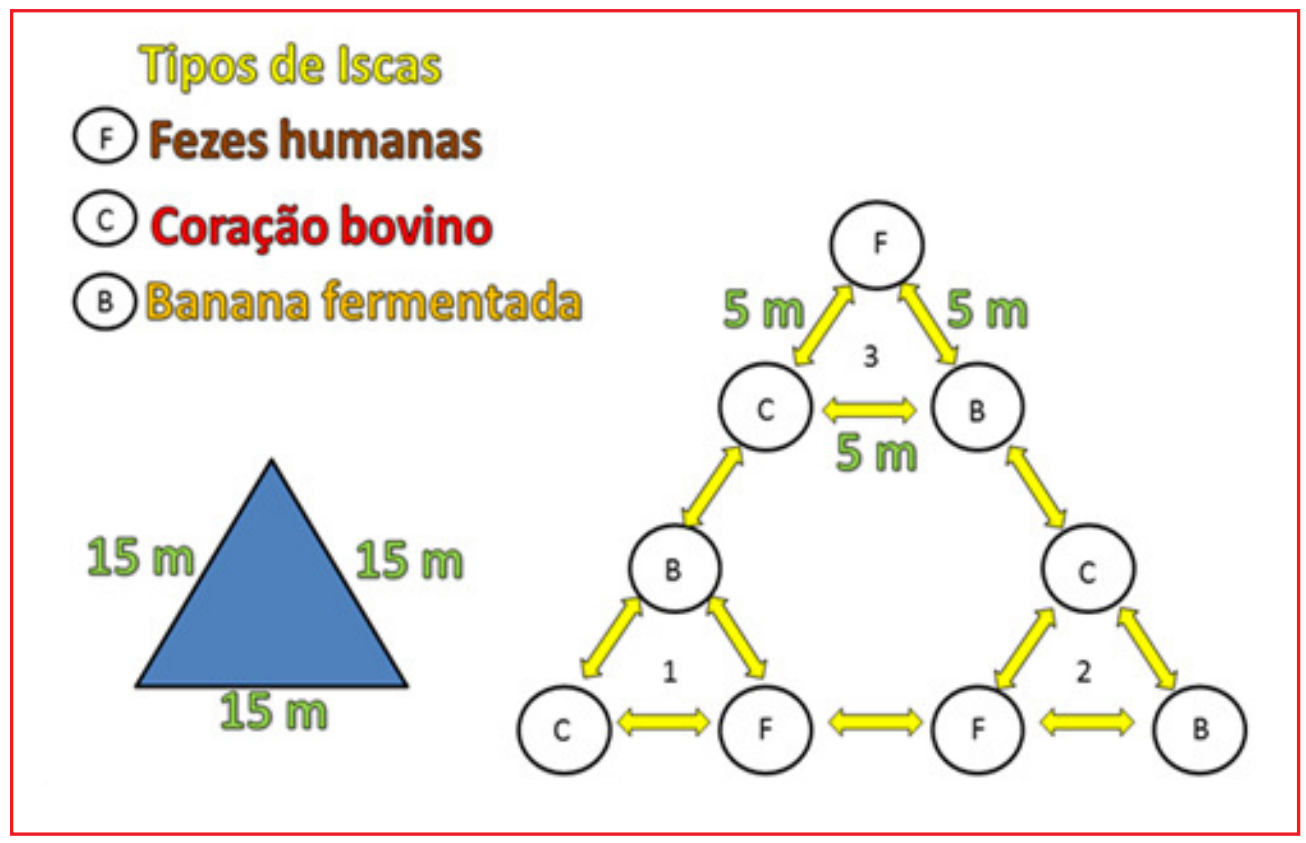

Figura 1 - Esquema da distribuição das armadilhas pitfall no interior do remanescente florestal, sendo cada círculo correspondente a uma armadilha pitfall.

equilátero de $15 \mathrm{~m}$ de lado (Figura 1). As armadilhas foram instaladas a aproximadamente cinco metros da bordadura do remanescente florestal.

A armadilha pitfall consiste de um recipiente de boca larga enterrado no solo de maneira que a abertura fique ao nível da superfície do mesmo (Leivas et al., 2013). As pitfalls consistiram em copos plásticos de $700 \mathrm{~mL}$ enterrados até o nível superficial do solo. No interior de cada armadilha era adicionado $250 \mathrm{~mL}$ de solução conservante (formaldeído a 1\%), com algumas gotas de detergente líquido neutro, para quebrar a tensão superficial da água, conforme metodologia utilizada por Santos e Cividanes (2007).

Os atrativos alimentares eram suspensos por um fio de cobre, em recipientes plásticos, nos quais eram inseridos aproximadamente $20 \mathrm{~g}$ do atrativo alimentar (Figura 2A e 2B). Foram utilizados três tipos de atrativos alimentares nesse estudo (fezes humanas, coração bovino em decomposição e banana fermentada) (Silva et al., 2007). Um rodízio de atrativos
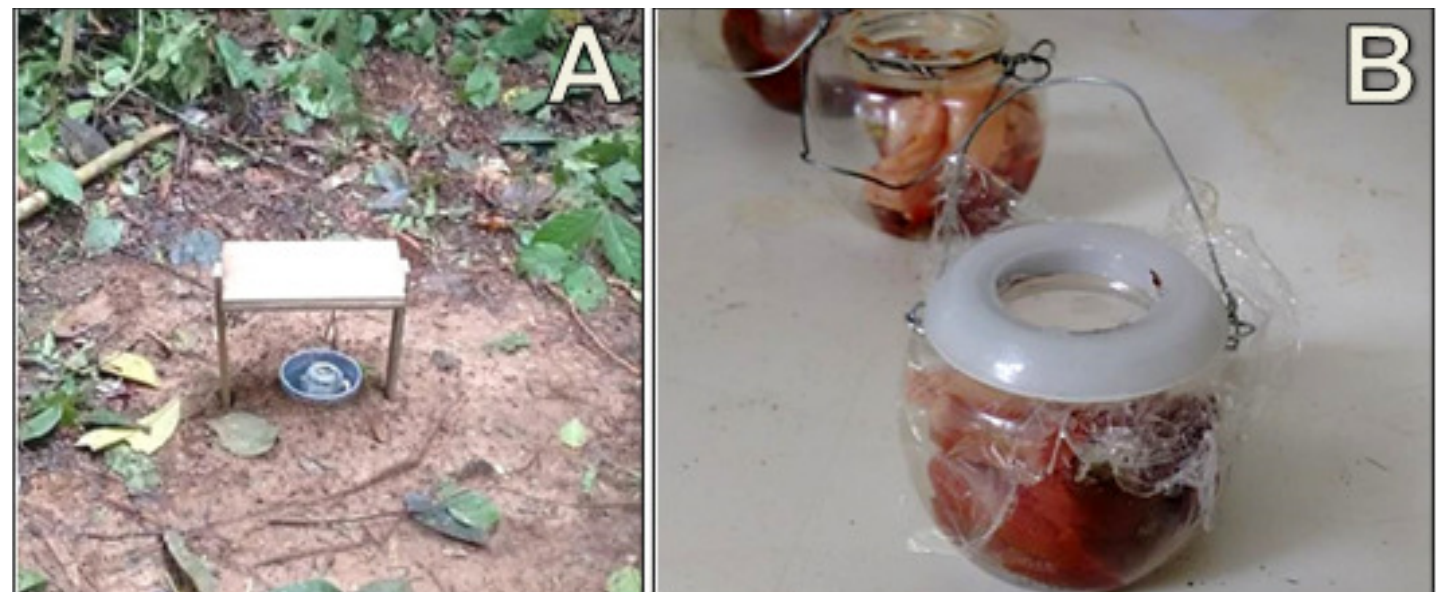

Figura 2 - Detalhe de armadilha pitfall iscada, com sua respectiva proteção de madeira(A) e do pote plástico contendo coração bovino em seu interior (B). (Crédito das fotos: Weidson Plauter Sutil). 
alimentares entre as pitfalls era realizado a cada coleta, a fim de reduzir a interferência da posição da armadilha em detrimento ao tipo de atrativo.

Sobre cada pitfall foi instalada uma cobertura quadrada de madeira $\left(12 \mathrm{~cm}^{2}\right)$, a fim de evitar o transbordamento pela água da chuva e/ou o entupimento das armadilhas por folhas e/ou detritos (Figura 2A).

A cada amostragem, o material coletado era levado até o Laboratório de Entomologia da Embrapa Acre, onde se procedia a triagem, com auxílio de peneira granulométrica. Os coleópteros pertencentes às famílias Histeridae, Staphylinidae e Scarabaeidae foram morfotipados, sob microscópio estereoscópio, e preservados em frascos contendo álcool a $70 \%$. Posteriormente, os escarabeídeos foram enviados ao taxonomista Dr. Paschoal Coelho Grossi (Universidade Federal Rural de Pernambuco - UFRPE, Recife, PE), para identificação ao menor nível taxonômico possível.

\section{Resultados e Discussão}

Foram coletados 6.601 besouros pertencentes às famílias Histeridae, Staphylinidae e Scarabaeidae, sendo essa última família a mais abundante em todo o período de estudo. Os escarabeídeos pertenciam a cinco tribos, 12 gêneros e 40 espécies (Tabela 1).

As tribos Coprini e Canthonini foram as melhores representadas com 15 e 14 espécies respectivamente, seguidas por Phanaeini (6 espécies), Onthophagini (3) e Oniticelini (2).

Coprini corresponde a uma das tribos mais ricas com gêneros que ainda são pouco estudados, em especial Canthidium e Dichotomius, os quais se encontram em processo de revisão sistemática. Estes grupos possuem os mais variados hábitos alimentares, encontrando-se preferencialmente em floresta primária e secundária (Korasaki et al., 2012). Os gêneros mais frequentes desta tribo foram Canthidium e Dichotomius com sete espécies, seguidos por Canthon com cinco espécies. Uma possível hipótese para justificar a maior abundância de Coprini neste levantamento é o fato de o fragmento de floresta corresponder a uma mata primária.

Canthidium é um grande gênero, com 153 espécies descritas, sendo a maior parte copro-necrófagas que vivem em florestas ou savanas neotropicais, fator que pode explicar a sua maior ocorrência (Vaz-de-Mello,
Tabela 1 - Espécies de Scarabaeidae (Coleoptera) coletadas em armadilhas pitfall iscadas, em remanescente florestal, no município de Rio Branco, Acre, de março a agosto de 2016

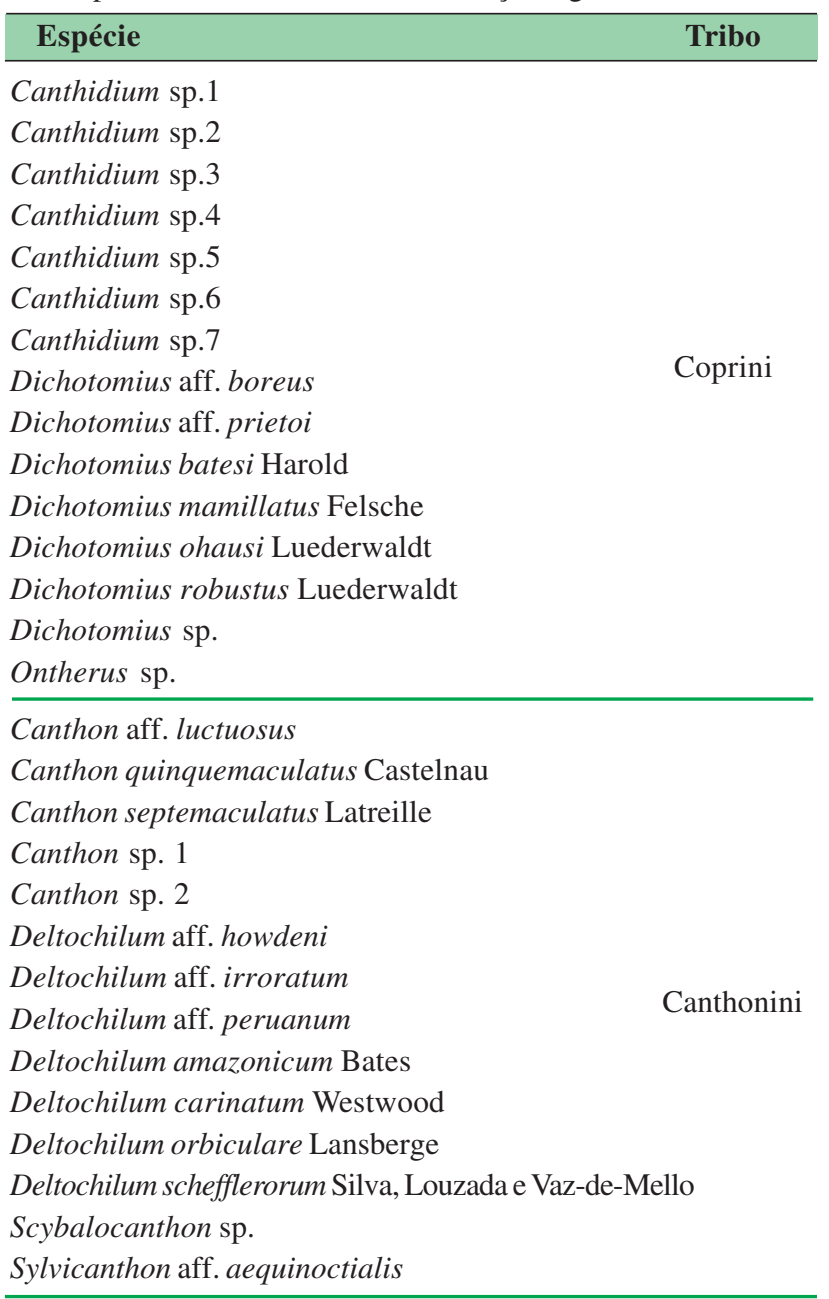

Oxysternon conspicillatum Weber

Oxysternon silenus Castelnau

Phanaeus bispinus Bates

Phanaeus camberforti Arnaud Phanaeini

Coprophanaeus aff. degallieri

Coprophanaeus telamon Erichson

Onthophagus aff. xanthomerus

Onthophagus haematopus Harold

Onthophagini

Onthophagus sp.

Eurysternus aff. hypocrita

Eurysternus caribaeus Herbst

Oniticelini

1999). Possuem hábitos alimentares generalistas, sendo atraídas por fezes humanas, além de carnes e frutas em decomposição. Há espécies indicadoras de ecossistemas florestais mais conservados, bem como outras, encontradas em ecossistemas perturbados 
(Silva et al., 2011). Sete morfoespécies de Canthidium já haviam sido coletadas em levantamento realizado por Rufino et al. (2016), utilizando armadilhas pitfall não iscadas, em remanescente florestal, no município de Plácido de Castro, AC, reforçando que esse gênero de Scarabaeidae é comum em ambientes florestais no estado do Acre.

Canthonini é uma das mais antigas e maiores tribos de Scarabaeidae apresentando vários hábitos alimentares, preferencialmente copro-necrófagos, mas que também utilizam frutos em decomposição e fungos como recurso alimentar (Vaz-de-Mello, 1999). Os gêneros mais frequentes foram Deltochilum com sete espécies, seguido por Canthon com cinco espécies. As espécies Deltochilum schefflerorum Silva, Louzada e Vaz-de-Mello e Oxysternon silenus Laporte, já haviam sido registradas no estado do Acre (Rufino et al., 2016).

Em trabalho similar, desenvolvido no estado do Acre por Vaz-de-Mello (1999), utilizando armadilhas pitfall iscadas (fezes humanas e carcaça de peixe), armadilha de interceptação de voo (Malaise) e coleta manual (rede entomológica), foram registradas 36 espécies de escarabeídeos, quatro a menos em relação a este estudo, havendo uma significativa diferença entre as espécies capturadas em ambos os trabalhos. Esse resultado aponta que, para se realizar um amplo levantamento de besouros escarabeídeos, fazem-se necessárias várias formas de captura, tais como: armadilhas iscadas, de interceptação de voo e coleta manual.

De acordo com Silveira Neto et al. (1995), os insetos de solo podem ser bons indicadores ecológicos, para a avaliação do impacto ambiental que venha a ocorrer em determinada região, já que a interação da comunidade biótica com o solo tem um papel vital na produção e manutenção da sua qualidade (Aquino, 1999). Segundo Rodrigues e Marchini (1998) os besouros coprófagos são utilizados no controle biológico da mosca-do-chifre, Haematobia irritans (L.) (Diptera: Muscidae), uma das principais pragas de bovinos no Brasil (Honer e Gomes, 1990). O escarabeídeo africano, Digitonthophagus gazela (Fabricius), foi introduzido no Brasil em 1989, para fins de controle biológico de moscas e incorporação de matéria orgânica no solo (Miranda et al., 1998; Koller et al., 2006).

No âmbito geral, verificou-se que o maior número de coleópteros foi capturado pelas armadilhas que continham o atrativo de coração bovino em decomposição (2.418 indivíduos capturados), seguido por fezes humanas (2.338) e banana fermentada (1.845), sendo o mais recomendado para captura de espécies de escarabeídeos de hábito copro-necrófago. A maior incidência de besouros nos dois primeiros tipos de atrativos se deve ao fato de a maioria dos representantes de Scarabaeidae possuir o hábito copronecrófago (Silva e Di Mare, 2012).

Segundo Silva et al., 2011, Canthon aff. luctuosus é capturada em fezes humanas, carnes e frutas apodrecidas. Canthon quinquemaculatus possui hábito necrófago e se adapta bem a ambientes perturbados. Eurysternus cariabaeus é considerada coprófaga, embora também seja atraída por frutas em decomposição.

Canthon quinquemaculatus Castelnau, Canthon septemaculatus Latreille, Deltochilum carinatum Westwood, Deltochilum orbiculare Lansberge, Dichotomius batesi Harold, Phanaeus bispinus Bates e Phanaeus camberforti Arnaud, representam primeiros registros de ocorrência no estado do Acre.

\section{Conclusões}

Devido à escassez de trabalhos com Coleoptera realizados na Amazônia Ocidental, especialmente no estado do Acre, este trabalho contribuiu para aumentar o conhecimento acerca da fauna de escarabeídeos ocorrentes no Estado. Pelo número de espécies capturadas nesse levantamento e, pelo hábito alimentar de algumas espécies identificadas, verifica-se que o remanescente florestal possui baixo impacto antrópico, com a maioria das espécies encontradas não tendo hábitos alimentares generalistas.

\section{Literatura Citada}

AAQUiNO, A. M. 1999. Meso e macrofauna do solo e sustentabilidade agrícola: perspectivas e desafios para o século XXI. In: Congresso Brasileiro de Ciência do Solo, Brasília, 1999. Anais. Brasília, DF, Sociedade Brasileira de Ciência do Solo.

BORGES, L. O. 2006. Estudo comparativo de assembléias de Histeridae e Staphylinidae (Insecta, Coleoptera) em áreas de mata semicaducifólia tropical e pastagem no Campus Samambaia, Goiânia, Goiás, Brasil. Revista de Biologia Neotropical (Brasil) 3(2):189-190. 
FAVILA, M. E.; HALFFTER, G. 1997. The use of indicator groups for measuring biodiversity as related to community structure and function. Acta Zoológica Mexicana 72:1-25.

FUNDAÇÃO DE TECNOLOGIA DO ESTADO DO ACRE FUNTAC. 1989. Inventário florestal e diagnóstico da regeneração natural da área do Programa de Desenvolvimento Rural Integrado do Estado do Acre - PDRI/AC. Rio Branco, AC, FUNTAC/INPA. 151p.

HALFFTER, G.; FAVILA, M. E. 1993. The Scarabaeinae (Insecta: Coleoptera) an animal group for analyzing, inventorying and monitoring biodiversity in tropical rainforest and modified landscapes. Biology International 27:15-21.

HALFFTER, G.; MATTHEWS, E. G. 1966. The natural history of dung beetles of the subfamily Scarabaeinae (Coleoptera: Scarabaeidae). Florida Entomologist 12:1-312.

HONER, M. R.; GOMES, A. 1990. O manejo integrado de mosca dos chifres, berne e carrapato em gado de corte. Campo Grande, EMBRAPA GADO DE CORTE. Circular Técnica, n. 22. 60p.

KOLLER, W. W., GOMES, A.; RODRIGUES, S. R. 2006. Perspectivas de degradação de fezes bovinas pelo besouro coprófago africano, Digitonthophagus gazella e espécies sulamericanas (Coleoptera, Scarabaeidae e Aphodiidae). Campo Grande, MS, EMBRAPA GADO DE CORTE . Comunicado Técnico, n. 100. 7p.

KORASAKI, V. et al. 2012. Taxocenose de Scarabaeinae (Coleoptera: Scarabaeidae) em Benjamin Constant, AM. Acta Amazonica (Brasil) 42 (3):423-432.

LEIVAS, F. W. T.; GROSSI, P. C.; ALMEIDA, L. M. 2013. Histerídeos (Staphyliniformia: Coleoptera: Histeridae) dos Campos Gerais, Paraná, Brasil. Biota Neotropica (Brasil) 13 (2):196-204.

LOBO, J. M.; MARTÍN-PIERA, F.; VEIGA, C. M. 1988. Las trampas pitfall con sebo, sus posibilidades en el estudio de las comunidades coprófagas de Scarabaeoidea (Col.). I. Características determinantes de su capacidad de captura. Revue D'ecologie et de Biologie du Sol 25 (1):77-100.

MELO, F. V. et al. 2009. A importância da meso e macrofauna do solo na fertilidade e como bioindicadores. Boletim Informativo da Sociedade Brasileira de Ciência do Solo (Brasil) 34 (1):39-43.

MILHOMEM, M. S.; VAZ-DE-MELLO, F. Z.; DINIZ, I. R. 2003. Técnicas de coleta de besouros copronecrófagos no Cerrado. Pesquisa Agropecuária Brasileira (Brasil) 38 (11): 1249-1256.

MIRANDA, C. H. B.; SANTOS, J. C. C.; BIANCHIN, I. 1998. Contribuição de Onthophagus gazella à melhoria da fertilidade do solo pelo enterrio de massa fecal bovina fresca. 1. Estudo em casa de vegetação. Revista Brasileira Zootecnia (Brasil) 27(5):681-685.

OLIVEIRA, M. V. N.; BRAZ, E. M. 1998. Manejo florestal em regime de rendimento sustentado aplicado à floresta do Campo Experimental da EMBRAPA/CPAF/AC. Rio Branco, AC, EMBRAPA ACRE. Boletim de Pesquisa, n. 21. $45 \mathrm{p}$.

Agrotrópica 30(1) 2018
OLIVEIRA, M. V. N. 1994. Composição florística e potenciais madeireiro e extrativista em uma área de floresta no estado do Acre. Rio Branco, AC, EMBRAPA ACRE . Boletim de Pesquisa, n. 9. 42p.

RODRIGUES, S. R.; MARCHINI, L. C. 1998. Besouros coprófagos (Coleoptera: Scarabaeidae) coletados em Piracicaba, São Paulo. Scientia Agricola (Brasil) 55(1):53-58.

ROSENBERG, D. M.; DANKS, H. V.; LEHMKUHL, D. M. 1986. Importance of insects in environmental impact assessment. Environmental Management 10(6):773-783.

RUFINO, C. P. B. et al. 2016. Escarabeídeos capturados em armadilhas pitfall em Plácido de Castro, AC. In: Congresso Brasileiro de Entomologia, 26, Maceio, AL. Anais. Maceio, AL, Sociedade Brasileira de Entomologia.

SANTOS, R. S.; CIVIDANES, F. J. 2007. Registro de opiliões (Arachnida: Opiliones) em três agrossistemas e remanescente florestal. Ecossistema (Brasil) 32 (1):9-12.

SAMPAIO, J. A. 2010. Levantamento e grupos tróficos de coleópteros cursores de solo em Sergipe: Importância dos coleópteros como indicadores do processo de recuperação florestal. Dissertação Mestrado. São Cristóvão, SE, Universidade Federal de Sergipe. 38p.

SILVA, F. A. B. et al. 2007. Comunidade de escarabeíneos (Coleoptera, Scarabaeidae) copro-necrófagos da região de Brejo Novo, Caruaru, Pernambuco, Brasil. Revista Brasileira de Entomologia (Brasil) 51(2):228-233.

SILVA, P. G.; VAZ-DE-MELLO, F. Z.; DI MARE, R. A. 2011. Guia de identificação das espécies de Scarabaeinae (Coleoptera: Scarabaeidae) do município de Santa Maria, Rio Grande do Sul, Brasil. Biota Neotropica (Brasil) 11 (4):329-345.

SILVA, P. G.; DI MARE, R. A. 2012. Escarabeíneos copronecrófagos (Coleoptera, Scarabaeidae, Scarabaeinae) de fragmentos de Mata Atlântica em Silveira Martins, Rio Grande do Sul, Brasil. Iheringia, Série Zoologia 102(2): 197-205.

SILVEIRA NETO, S. et al. 1995. Uso da análise faunística de insetos na avaliação impacto ambiental. Scientia Agrícola (Brasil) 52(1):9-15.

SLIPINSKI, S. A.; LESCHEN, R. A. B. ; LAWRENCE, J. F. 2011. Order Coleoptera Linnaeus, 1758. In: Z. Q. Zhang ed. Animal biodiversity: An outline of higher-level classification and survey of taxonomic richness. Zootaxa 3148:203-208.

TEIXEIRA, C. C. L.; HOFFMANN, M.; SILVA-FILHO, G. 2009. Comunidade de Coleoptera de solo em remanescente de Mata Atlântica no estado do Rio de Janeiro, Brasil. Biota Neotropica (Brasil) 9(4):91-95.

VAZ-DE-MELLO, F. Z. 1999. Scarabaeidae s. str. (Coleoptera: Scarabaeoidea) de um fragmento de Floresta Amazônica no Estado do Acre, Brasil. 1. Taxocenose. Anais da Sociedade Entomológica do Brasil (Brasil) 28(3):439-446.

WINK, C. et al. 2005. Insetos edáficos como indicadores da qualidade ambiental. Revista de Ciências Agroveterinárias (Brasil) 4(1):60-71. 